\title{
A Modified ABC Algorithm \& Its Application to Wireless Sensor Network Dynamic Deployment
}

\author{
Alina Rakhi Ajayan, Prof. S. Balaji \\ Dept of ECE, DMI College of Engg, Chennai, India \\ Dept of ECE, DMI College of Engg, Chennai, India
}

\begin{abstract}
Wireless Sensor Networks plays a gargantuan role in instrumenting the modern day world; hence it is crucial to develop an optimal design flow. Out of the various Swarm intelligent algorithms developed so far, Artificial Bee Colony (ABC) algorithm offers a vivid scope for this objective. First introduced for solving numerical optimization problems, ABC has now spread across almost every phase of WSN construction. In this study, an improved $A B C$ algorithm has been proposed to match the different characteristics of wireless sensor network deployment process, which will be optimum for real time dynamic network functioning.

KEYWORDS: Wireless Sensor Networks, Swarm Intelligent Algorithms, scouts, foragers, onlookers, waggle dance, self organization, Opposition-based learning.
\end{abstract}

\section{INTRODUCTION}

Recent advances in sensing, computing and communication technologies coupled with the need to continuously monitor physical phenomena have led to the development of Wireless Sensor Networks (WSNs). A WSN is formed by deploying sensor nodes in an application area forming an infrastructure of sensor/measurement, computation, and communication elements to instrument, observe, and react to events and phenomena in a specified environment. In most real-time deployment solutions, they demand self-organizing capabilities to keep track of the dynamic domain. The main benefits of such a configuration is the spatial diversity they provide, enabling applications such as target detection \& tracking as it moves throughout the sensor field; weapon targeting and area denial Such networks are suitable for a wide variety of applications such as surveillance, precision agriculture, smart homes, automation, vehicular traffic management, habitat monitoring, disaster detection, etc. Smart disposable micro sensors can be deployed on practically any kind of environment - ground, air, under water, vehicles, living beings, buildings, \& volcanoes.

Designing groups of spatially distributed autonomous sensors, connected to each other forming a network topology (ad-hoc, Star, Mesh, etc), \& group wise to APs, require much precision and calculations. Most of the dynamic deployment solutions deal with optimum positioning through 1 or more metrics, starting from an initial random map. But, in such a case we might observe several demerits, like slower convergence, uncertainity in avoiding local minimums, overlap and redundancy, etc. As studied in [15], this can lead to added energy, delay and overhead expenses. Thus we resort to advanced deployment algorithms.

Bio-inspired principles have found their way into WSN R \& D due to the appealing analogies between biological systems and large network. The dynamics of many biological systems and the laws governing them are based on a surprisingly small number of simple rules, yielding collaborative \& effective mechanisms for resource management, task allocation, and synchronization without any central controlling element. Such Swarm Intelligent Algorithms give simple solutions to WSN Deployment problems. Artificial bee colony algorithm is one such

\section{THE ARTIFICIAL BEE COLONY ALGORITHM}

The Artificial Bee Colonies (ABC) is another novel optimization algorithm that comes under Swarm inspired by social behavior of natural bees. It is a complete analogy of the foraging activities of the honey bee colony, consisting of morphologically uniform individuals with different temporary specializations to represent a distributed adaptive system of smart control packets. These packets use limited computational and energy resources to explore the environment/network. They cooperate mutually \& efficiently by releasing information about the discovered paths and estimated quality at the specified nodes. Due to these similarities between foraging behaviors in insect societies and network routing, in the last decade, many SI based routing protocols, specially $\mathrm{ABC}$, have been developed for wired networks, satellite networks, MANETs, and, more recently, WSNs. The benefit of such organizations is increased flexibility to adapt to the changing environments.

The honey bee colonies symbolize design of optimization strategies for dynamic, time-varying, and multi-objective problems. The bee members utilize a sophisticated communication protocol with bee-to-bee signals and when required, \& Stigmergic feedback cues for bee-to-group or group-to-bee interaction. The main advantage from other swarm intelligent protocols like ant colony optimization is communications are based on 
visual activity \& path search, unlike backtracking in many others.

Worker bees typically do brood rearing for the first week, engage in other hive maintenance duties (wax secretion, guarding, undertaking, nectar processing), when they are middle-aged switch to foraging and when about three weeks old, to colony defense. Foraging is critical task for colony survival. Foragers constantly leave the hive searching for new sources of nutrient, return with nutrient samples, and try to recruit other bees to exploit the food site by competing with other site sample bearers. Foragers announce a food source of interest to their fellow foragers by doing a dance on the dance floor inside the hive termed Waggle Dance. It is a particular figure-eight dance symbolizing the direction of the food source in terms of angular position with the sun in reference, and the distance in the duration of each waggle-run. A very short distance will be a waggle dance resembling a round dance. Foragers prefer nearer food sites over distant ones in order to increase the net energetic efficiency of the colony. Nectar foragers use Tremble Dance to show a rich food source detection, yet no free employee, upon return to the hive, after a certain threshold time, This stimulates the bees inside the hive to increase and/or to switch to nectar processing activities, and to inhibit the outside foragers from recruiting additional bees, thus keep colony's nectar processing rate matched with its nectar intake rate. Stochastic selection of food sites enables optimal choice \& effective balancing between exploitation and exploration.

In $\mathrm{ABC}$ algorithm, colony bees are divided into three groups: employed bees, onlooker bees and scout bees. The number of employees is equal to the number of food sources i.e. number of solutions in the population.. The employee whose food source is exhausted becomes the scout bees, who also start the initial food foraging. Each food source position is equivalent to a potential solution of optimization problem, with its quality or fitness value as the base of selection, i.e. nectar amount. Whenever any bee finds the food, it signals the other bees by its dance stigmergy, the quantity and the location of the food source. This attracts a large number of bees towards good sources to search. Thus ABC algorithm model have three operational phases:

1. Scout bees do random search for the food, \& find near optimal food sources, completely random.

2. Employed bees visit food source and gather information about food source location and the quality. they have memory of the places they have visited before and quality of food there, \& performs the local search to try to exploit the neighboring sources to locate the best

3. Onlooker bees wait in the dance area to decide which food source is better on the basis of information provided by employees. They perform the global search for discovering the global optimum

4. The model has the following basic features to support self organization:

i) Positive feedback: As nectar amount of food sources increases, number of onlookers visiting them increases.

ii) Negative feedback: The exploitation process of poor food sources is stopped by waggle dance.

iii) Fluctuations: The scouts carry out a random search process for discovering new food sources.

iv) Multiple interactions: Bees share their information about food sources on the dance area.

\section{THE PROPOSED MODEL}

Generic ABC Algorithm pseudo code is given below, based random initial positioning:

1: Set swarm size S, dimensionality N, search space domain MI.

2: Create a population of $S$ random food sources and evaluate their fitness

3: REPEAT THE FOLLOWING STEPS.

4: Send employed bees to exploit the food sources

5: For each solution i determine a neighbor $\mathrm{k}$ and generate a new best solution.

6: Compute the probabilities for the solutions,

7: Send onlooker bees to exploit the food sources according to probabilities.

8: For each solution i determine a neighbor $\mathrm{k}$ and generate a new best solution.

9: Determine abandoned solutions and send scout bees to search new food sources to replace them.

10: Update best fitness and respective food source position

11: $\mathrm{MI}=\mathrm{MI}+1$

12: UNTIL MI = MaxMI.

As we know, this algorithm needs to be modified to fit into the optimization constraints and range. The initial positioning is made arbitrarily closer to an optimal positioning using Voronoi diagrams. The concept of 'greedy bee' helps make better selection trials. Greedy bees will always be attracted towards the flower patch having the maximum nectar or the most potential food source. This is useful in avoiding local troughs and minima.

An attractive concept that is applicable is the Opposition-based learning. It is an attempt made to generate better initial food sites for the bees, especially scouts' new locations. The search space is contracted to enable scouts to locate the food sources in a fewer number of cycles. The concept is: for a real number ' $x$ ' in the range $[a, b]$, i.e. $x \in[a, b]$, the opposite number " $x$ " " of $x$ is defined as $x$ ' $=a+b-x$. The bottom-line of this concept is that $50 \%$ of the computation time the current solution is further away from the optimum than its 
opposite solution. Hence considering both the numbers and retaining the better of the two, chances of finding the optimum quickly is improved.

Optimization with respect to network communication paradigms also helps in improving efficiency. The wireless links, system model, etc determine the energy parameters of a WSN, which is of course the designers' primary concern. Instead of complying to minimum no. of hops, distance, graph structures etc, inclusion of minimum energy threshold, remaining energy measure, energy-distance metric, etc, keeps a full check on the throughput, and lifetime as well.

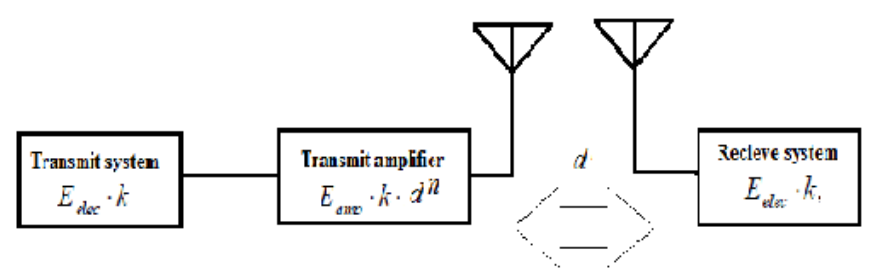

Figure 1. basic nodal transmission model

The proposed algorithm comprises of following steps:

\section{PSUEDOCODE}

1. Initialize Control Parameters of ABC Algorithm: Colony size CS, dimension of the problem $\mathrm{D}=2$, and Limit for scout $\mathrm{L}=(\mathrm{CS} * \mathrm{D}) / 2$.

2. Initialize scout bee positions as per subroutine below:

2.1. Let $\mathrm{s}$ be the total no. of scout bees positions.

2.2. Generate Voronoi diagram based positioning for the m nodes.

2.3. Evaluate the fitness of the positioning.

$f_{i}=d(m, n)=-\left[\left[\frac{c \sqrt{P_{t x}}}{4 \pi f}\right] \cdot \sqrt{\left[P_{r x}(m, n)\right]}\right]$.

For node $s_{i}(m, n)$, Where $m$ and $n$ are node position coordinates, $c=$ speed of light, Ptx $=$ transmitted signal power per node to reach the entire domain dimension, $\operatorname{Prx}=$ received signal strength, $f=$ channel frequency

2.4. Calculate weightage probability as,

$P_{i}=\left[\frac{\left(0.9 \times f_{i}\right)}{f_{\text {best }}}\right]+0.1$.

3. Set no. of iterations for optimisation, MaxIt.

4. REPEAT for $\mathrm{i}=1$ to MaxIt

4.1. Assign $\mathrm{k}$ employee bee nodes, best out of s scouts, as $\mathrm{k}_{\mathrm{i}}$.

4.2. Produce new solutions for onlooker positions based on Opposition learning, as $\mathrm{v}_{\mathrm{ij}}$, for each $\mathrm{k}_{\mathrm{i}}$.

4.3. Check for fitness as, use Probabilistic detection model to prevent coverage overlap.

$$
c_{m n}\left(v_{i j}\right)=\left\{\begin{array}{c}
0, \text { if } r+r_{e} \leq d\left(k_{i}, v_{i j}\right) \\
e^{\left(\frac{-\lambda_{1} \alpha 1^{\beta 1}}{\alpha 2^{\beta 2}+\lambda_{2}}\right)}, \text { if } r-r_{e} \leq d\left(k_{i}, v_{i j}\right) \leq r+r_{e} \\
1, \text { if } r+r_{e} \geq d\left(k_{i}, v_{i j}\right)
\end{array}\right.
$$

And

4.4. Apply greedy selection process between new nodes.

$\mathrm{v}_{\mathrm{ij}}=\mathrm{k}_{\text {best }, \mathrm{i}}+\mathrm{r}_{\mathrm{ij}}\left(\mathrm{k}_{\text {best }, \mathrm{i}}-\mathrm{k}_{\mathrm{i}}\right)$

4.5. Determine the probability values out of their fitness values.

4.6. Produce new solutions for $\mathrm{v}_{\mathrm{ij}}$ out of this check.

4.7. Record the best solutions yet.

4.8. Check for abandonment condition according to the remaining energy model,

$$
F_{i j}=\sum_{i}\left[\sum_{j}\left[d\left(v_{i j}, \mathrm{k}_{\mathrm{i}}\right)\right]^{2}+\left[d\left(v_{i j}, \mathrm{k}_{\mathrm{best}, \mathrm{i}}\right)\right]^{2}\right] \text {, }
$$

$\&$ if satisfied replace with new solutions using Opposition based learning.

4.9. Increment $\mathrm{i}=\mathrm{i}+1$. 


\subsection{UNTIL i = MaxIt.}

5. $\quad$ Ensure deployment coverage check using

$$
\mathrm{c}_{\mathrm{mn}}\left(\mathrm{v}_{\mathrm{ov}}\right)=1-\prod_{\mathrm{v}_{\mathrm{ij}}}\left(1-\mathrm{c}_{\mathrm{mn}}\left(\mathrm{v}_{\mathrm{ij}}\right)\right) \geq \mathrm{c}_{\mathrm{mnth}}
$$

\section{CONCLUSION \& FUTURE WORK}

Artificial Bee Colony algorithm has opened up a vast stage for WSN protocol suite design. Like any swarm intelligent scheme, they are compatible to any stage of a WSN design and implementation, which makes them an attractive choice as the base. Though the generic version gives inferior performance in high target goals, a few adaptations to fit into our formulated problem gives colorful results. This paper makes such a proposal, which has been molded to optimize the total deployment scenario

Further goals include adapting the same algorithm with minor parameter variations for the routing scheme. Hence it can, by itself, constitute the backbone of a complete WSN network layer protocol suite. The analysis part can be done in NS2 simulator, or even Matlab.

\section{REFERENCES}

[1] Fahad S. Abu-Mouti, Mohamed E. El-Hawary, "Overview of Artificial Bee Colony (ABC) Algorithm and Its Applications", 978-14673-0750-5/12/ @2012 IEEE

[2] Celal "OZT"URK, Dervi,s KARABO־GA, Beyza G"ORKEML", "Artificial bee colony algorithm for dynamic deployment of wireless sensor networks”, Turk J Elec. Eng \& Comp Sci., Vol.20, No.2, 2012, c_ T“UB'ITAK doi:10.3906/elk-1101-1030

[3] Gaige Wang 1,2, Lihong Guo 1,*, Hong Duan 3, Luo Liu 1,2 and Heqi Wang. "Dynamic Deployment of Wireless Sensor Networks by Biogeography Based Optimization Algorithm” J. Sens. Actuator Netw. 2012, 1, 86-96; doi:10.3390/jsan1020086,Journal of Sensor and Actuator NetworksISSN 2224-2708.

[4] 4 Siba K. Udgata, Samrat L. Sabat, S. Mini , "Sensor Deployment in Irregular Terrain Using Artificial Bee Colony algorithm", 9781-4244-5612-3/09/\$26.00_c 2009 IEEE

[5] Avinash Kaur, Sonu Agrawal, Department of CSE, CSVTU, Bhilai, India , "Locality Detection in Wireless Sensor Network using Population based Algorithm”, International Journal of Computer Applications (0975 - 8887) Volume 51- No.4, August 2012.

[6] Zhang Dongli, Guan Xinping, Tang Yinggan, Tang Yong, "Modified artificial bee colony algorithms for numerical optimization", IEEE, 2012

[7] Dervis Karaboga1, Selcuk Okdem, and Celal Ozturk, "Cluster Based Wireless Sensor Network Routings using Artificial Bee Colony Algorithm”, 978-1-4244-7107-2/10/\$26.00 @2010 IEEE

[8] Selcuk Okdem, Dervis Karaboga and Celal Ozturk, "An Application of Wireless Sensor Network Routing based on Artificial Bee Colony Algorithm",

[9] Celal Ozturk *, Dervis Karaboga and Beyza Gorkemli, " Probabilistic Dynamic Deployment of Wireless Sensor Networks by Artificial Bee Colony Algorithm", Sensors 2011, 11, 6056-6065; doi:10.3390/s110606056, ISSN 1424-8220, 3 June 2011.

[10] Tarun Kumar Sharma, Millie Pant, "Enhancing the Food Locations in an Artificial Bee Colony Algorithm ",978-1-61284-0529/11/\$26.00 @2011 IEEE proceedings.

[11] Dervis Karaboga, Beyza Gorkemli, "A Quick Artificial Bee Colony -qABC- Algorithm for Optimization Problems", Research Fund of the Erciyes University, Project Number: FBA-11-3536, 978-1-4673-1448-0/12/\$31.00 @2012 IEEE proceedings.

[12] Bilal Babayigit, Resul Ozdemir, "A modified artificial bee colony algorithm for numerical function optimization", 978-1-46732713-8/12/\$31.00 @2012 IEEE proceedings.

[13] Wei-Ping Lee, Wan-Ting Cai, “A Novel Artificial Bee Colony Algorithm with Diversity Strategy”, 2011 Seventh International Conference on Natural Computation, 978-1-4244-9953-3/11/\$26.00 (C2011 IEEE proceedings.

[14] 15. ISSN 1943-3581, Network Protocols and Algorithms, 2010, Vol. 2, No. 2,Macro Institue, Coverage in Wireless Sensor Networks: A Survey, Raymond Mulligan, Habib M. Ammari.

[15] 16. Hosny M. Ibrahim, Nagwa M. Omar, Ali H. Ahmed, “ IMPROVING WIRELESS SENSOR NETWORKS PERFORMANCE BY USING CLUSTERED VIRTUAL RINGS”, International Journal of Ad hoc, Sensor \& Ubiquitous Computing (IJASUC) Vol.3, No.3, June 2012, DOI : 10.5121/ijasuc.2012.3304. 